\title{
Mujeres lesbianas en el movimiento LGBTI+ chileno. Motivación y experiencia $^{1}$
}

\author{
Catalina Otárola Arriagada ${ }^{2}$ \\ Catalina Tapia Pizarro ${ }^{3}$
}

Fecha de recepción: 16 de agosto de 2017

Fecha de aprobación: 25 de enero de 2018

\begin{abstract}
Resumen
La investigación contenida en este artículo nace de la inquietud acerca de la participación de las mujeres lesbianas en el movimiento LGBTI+ chileno. Considerando el género como un factor que otorga al individuo una posición de poder determinada respecto de otros y que, además, media en la experiencia de este individuo, tanto en la sociedad como en el movimiento, se analizaron las motivaciones de las mujeres lesbianas para participar en el movimiento LGBTI+ y sus experiencias al interior del mismo como elementos clave en el desarrollo de su participación. Con este objetivo en mente, se entrevistó a diez mujeres lesbianas que trabajaban activamente en distintas organizaciones-miembro del movimiento LGBTI+ chileno (Fundación Iguales, MOVILH, MUMS y Rompiendo el Silencio).
\end{abstract}

Palabras clave: mujeres lesbianas, LGBTI, género, diversidad sexual, movimientos sociales.

\section{Lesbians in the chilean LGBTI + movement. Motivation and experience}

\begin{abstract}
The research in this article is based on the concern about the participation of lesbians in the Chilean LGBTI + movement. Considering gender as a factor giving the individual a position of specific power in relation to others and that is important in the experience of that individual, both in society and in the movement, it analyzes motivation of lesbian women to participate
\end{abstract}

\footnotetext{
Los resultados contenidos en este artículo corresponden a una tesis para obtener el grado de Licenciada en Sociología en la Universidad Diego Portales. La investigación fue realizada durante 2015 y ya se encuentra finalizada.

2 Socióloga, licenciada de la Universidad Diego Portales, Santiago, Chile. Contacto: c.otarola.arriagada@gmail.com

3 Socióloga, licenciada de la Universidad Diego Portales, Santiago, Chile. Contacto: catalina.tpizarro@gmail.com
} 
in the LGBTI + movement and the experiences within as key elements in the development of their participation. With this in mind, we interviewed ten lesbians who worked actively in different organizations members of the Chilean LGBTI + movement (Fundación Iguales, MOVILH, MUMS and Rompiendo el Silencio.)

Key words: Lesbian, LGBTI, gender, sexual diversity, social movements.

\title{
Mulheres lésbicas no movimento LGBTI+ chileno. Motivação e experiência
}

\begin{abstract}
Resumo
A pesquisa contida neste artigo nasce da preocupação perante a participação das mulheres lésbicas no movimento LGBTI+ chileno. Considerando o gênero como um fator que confere ao indivíduo uma posição de poder determinada a respeito de outros e que, além disso, medeia na experiência deste indivíduo, tanto na sociedade quanto no movimento, foram analisadas as motivações das mulheres lésbicas para participar no movimento LGBTI+ e as suas experiências ao interior dele como elementos-chave no desenvolvimento da sua participação. Com este objetivo na mente, foram entrevistadas dez mulheres lésbicas que trabalhavam ativamente em diferentes organizaçõesmembro do movimento LGBTI+ chileno (Fundación Iguales, MOVILH, MUMS e Rompiendo el Silencio).
\end{abstract}

Palavras-chave: mulheres lésbicas, LGBTI, gênero, diversidade sexual, movimentos sociais.

\section{Introducción}

El movimiento por la diversidad sexual en Chile se posiciona con fuerza en la década del 80 del siglo pasado, con un discurso principalmente apuntado a los efectos que el VIH-SIDA tenía sobre la comunidad homosexual. Fueron varias las organizaciones que nacieron con posterioridad a los años de la dictadura militar, como es el caso del Movimiento de Liberación Homosexual ("MOVILH Histórico") en los años noventa, de la mano de importantes activistas homosexuales, como Juan Pablo Sutherland y Rolando Jiménez. A través de estas organizaciones, el movimiento LGBTI ${ }^{4}$ chileno problematizó cuestiones como la discriminación sufrida por la diversidad sexual y consiguió grandes avances, como la

4 La sigla LGBTI+ representa a lesbianas, gays, bisexuales, trans e intersexuales, y el signo "+" se utiliza para incorporar al resto de las identidades que pueden ser consideradas dentro de esta sigla, como por ejemplo asexuales, pansexuales, etc. 
modificación del artículo 265 del Código Penal, el cual castigaba la sodomía consentida entre hombres adultos (Robles, 2008).

Sin embargo, el movimiento no estuvo exento de disputas y quiebres, que llevaron a que el trabajo en conjunto se dificultara y desgastara, lo que luego se tradujo en la división definitiva del "MOVILH Histórico", surgiendo otras organizaciones, como el "Movimiento Unificado de Minorías Sexuales" (MUMS), constituido como el "heredero político" de esta organización y que realizó un fuerte trabajo en materia de Derechos Humanos y VIH, hasta la actualidad (MUMS, s.f.). En 1999 se funda, de la mano de Rolando Jiménez, otra de las organizaciones emblemáticas del movimiento LGBTI+ chileno, el Movimiento de Integración y Liberación Homosexual (MOVILH), que orientó su trabajo hacia el diseño de "acciones jurídicas, legislativas, culturales, sociales y económicas destinadas a la erradicación de la violación a los Derechos Humanos" de las minorías sexuales" (MOVILH, s.f.).

Con el paso del tiempo, el movimiento LGBTI+ continuó creciendo a través de la fundación de más organizaciones, lo que se tradujo en la ampliación de sus horizontes de lucha y un robustecimiento de sus propuestas. Dentro de estas organizaciones se encuentra "Rompiendo el Silencio", fundada en 2002 por la activista Erika Montecinos y conformada solo por mujeres lesbianas, la cual busca generar una "mayor incidencia pública de las lesbianas en el ámbito político activista" . Otra organización que ha logrado posicionarse como un referente del movimiento LGBTI+ es "Fundación Iguales". Ésta nace en 2011, por iniciativa de Luis Larraín, Pablo Simonetti y Antonio Bascuñán, y tiene como misión "trabajar a través de la investigación, la difusión, la educación, el desarrollo de Políticas Públicas y la gestión legislativa, para conseguir la plena inclusión de la diversidad sexual en la sociedad chilena" (Fundación Iguales, s.f.).

Si bien se puede hablar de movimiento LGBTI+, puesto que existen metas y objetivos a largo plazo en común, hay diferencias 
en los medios para alcanzar esos objetivos y en el énfasis que se da a ciertas problemáticas, acentuando, en mayor medida, ciertas identidades respecto a otras, por ejemplo, las identidades masculinas homosexuales. Las distintas desigualdades sociales (clase, género, etc.) se mantienen y reproducen al interior del movimiento, al mismo tiempo que se relacionan entre sí, produciendo experiencias propias de cada individuo y generando jerarquías de género, sexualidad e identidad de género dentro del propio movimiento, por nombrar algunas dimensiones.

Considerando lo anterior, resulta de interés preguntarse por la participación que las mujeres tienen al interior de este movimiento, que en su historia ha estado liderado principalmente por hombres homosexuales. Especialmente surge la pregunta por la forma en que el movimiento "interioriza" y vive, en lo cotidiano de sus organizaciones, la búsqueda de la igualdad y la acepción de la diversidad en relación a sus distintos miembros. Preguntas como de qué forma se relacionan las distintas identidades del movimiento o si hay una efectiva reproducción de la posición subordinada de las mujeres al interior de las distintas organizaciones guían esta investigación. En este sentido, este artículo analiza las motivaciones y experiencias que las mujeres lesbianas tienen al interior de distintas organizaciones del movimiento LGBTI+ chileno, dando cuenta de que el género de los individuos se constituye como un factor mediador de éstas.

\section{Marco conceptual}

\section{Motivaciones}

El concepto de "motivación" se relaciona con aquello que lleva a un sujeto determinado a tomar la decisión de, en este caso, participar de una organización en particular. En este sentido, los planteamientos de Honneth acerca de la "lucha por el reconocimiento" que llevan a cabo los sujetos en el contexto de un movimiento social, están estrechamente relacionados con sus moti- 
vaciones para llevar adelante aquellas luchas. Los motivos para la rebelión y la resistencia estarían constituidos por experiencias morales de menosprecio vividas por los sujetos, las cuales son consecuencia de que sus expectativas de reconocimiento son defraudadas e incumplidas -entendiéndose aquel reconocimiento como un reconocimiento social, en el que el sujeto puede saberse a sí mismo respetado en su entorno sociocultural (Honneth, 1997)-. Así, aquellas experiencias de menosprecio entorpecen la formación personal de la identidad de los sujetos.

Es necesaria, como condición del surgimiento de un movimiento, la existencia de una "semántica colectiva" que permita interpretar las experiencias personales de menosprecio como algo que concierne al colectivo de sujetos en su conjunto (Honneth, 1997). Así, las experiencias que las mujeres lesbianas pudiesen haber vivido a lo largo de su vida, ya sean episodios de violencia, discriminación, etc., se constituyen como motivaciones para participar en el movimiento LGBTI+, siempre en miras a ciertos objetivos que, si bien hacen referencia a la subjetividad de las mujeres, se configuran también como aquel "espacio intersubjetivo" en que aquellas experiencias comienzan a ser colectivas y apelan al movimiento LGBTI+ en su conjunto. De esta manera, la motivación tendría sus raíces en las diferentes psicologías individuales de los sujetos y en sus personalidades, pero se consolida en la interacción con otros (Melucci, 1999). No obstante, la motivación de los sujetos no tendría que ver exclusivamente con la persecución de ciertos fines racionales, sino que sus emociones y valores también se establecen como el "pegamento de la solidaridad" y lo que moviliza el conflicto (Collins, 1990, citado en Jasper, 1998). Ciertos afectos y emociones como la ira, la solidaridad, la indignación, la compasión, el entusiasmo, entre otros, pueden guiar a la gente hacia los movimientos sociales, mantenerlos dentro de ellos e, incluso, alejarlos (Jasper, 1998). En este sentido, y siguiendo los planteamientos de Gould, "las emociones son inseparables de los procesos políticos: moldean las nociones de la gente sobre lo que es políticamente posible o deseable" (2004, citado en Latorre, 2005, p.43). 
Lo que sienten las mujeres lesbianas, sus emociones y sentimientos, lejos de estar separado del trabajo político que ellas realizan, se constituye como motivación para la participación en el movimiento LGBTI+ y, al mismo tiempo, como aquello que las mantiene realizando aquel trabajo. El foco en las motivaciones de las mujeres lesbianas no solo permite entender el porqué de su participación y cómo se ha desarrollado su experiencia dentro del movimiento, sino que también entrega un panorama de lo que les moviliza dentro del movimiento LGBTI+ chileno.

\section{Experiencia}

En torno a este concepto, Dubet indica que las experiencias, emociones y acciones de los individuos son sociales y que "no se podrían comprender independientemente de los sistemas de relaciones y de representaciones que los 'fabrican'" (2011, p.58), es decir, las experiencias y motivaciones de las mujeres en el movimiento no se pueden separar del contexto social en el que se desarrollan, siendo afectadas por él, de manera que características como el género y las relaciones entre individuos, que surgen por su efecto, tendrán un rol importante en marcar las experiencias de los individuos, en este caso de mujeres lesbianas participantes del movimiento LGBTI+.

Para dar cuenta de esta relación entre exterioridad e interioridad, Dubet desarrolla el concepto de "experiencia social", que se constituye como "la cristalización (...) de lógicas de acciones diferentes" (Dubet, 2011, p.117) que los individuos deben administrar con el "fin de constituirse como sujetos" (Ibíd.). Considerando específicamente la cuestión del género se encuentra el trabajo de Teresa de Lauretis, quien indica que la experiencia es:

Un complejo de efectos de significado, hábitos, disposiciones, asociaciones y percepciones, resultantes de la interacción semiótica del yo y del mundo externo. La constelación o configuración de efectos de significados cambia 
y es reformada continuamente para cada sujeto con un compromiso continuo con la realidad social, una realidad que incluye -y para las mujeres, centralmente- las relaciones sociales de género. (De Lauretis, 1989, p.19)

Si bien, como indica Cháneton, hablar de una interacción entre un yo y el mundo puede resultar problemático, "ya que presupone un sujeto previo a la cultura que sería luego moldeado por ésta" (2099, p.81), la definición de De Lauretis refuerza la idea de Dubet de la relación de la subjetividad con el sistema social y cultural, y explicita la importancia del género en el desarrollo de la experiencia.

\section{Género y poder}

Las temáticas del género y del feminismo han estado adquiriendo cada vez más fuerza y validez al interior del mundo académico y la sociedad en general, abarcando más ámbitos de investigación e intervención. Siguiendo a Kirkwood (2017), este surgimiento y posicionamiento de la temática femenina puede situarse en los cambios científicos y tecnológicos de los últimos tiempos, que han permitido desechar las "consecuencias de los roles biológicos" asociadas a los géneros, por ejemplo:

El control de la natalidad y los métodos de alimentación artificial permiten que las parejas puedan elegir cuándo tener hijos y quién los alimentará; hay un avance personal para las mujeres (salen del hogar) y existe una importancia cultural potencial para ambos sexos: pueden compartir el trabajo y la alegría de criar niños (el hombre vuelve al hogar). (Kirkwood, 2017, p. 20)

A pesar de lo anterior, las valorizaciones y significaciones asignadas a cada género no han cambiado a la misma velocidad que lo han hecho estos procesos tecnológicos, por lo que cabe preguntarse qué mecanismos culturales y sociales sostienen estas significaciones y las desigualdades asociadas a ellas. 
Por otro lado, Kirkwood (2017), citando a Beauvoir, hace referencia a la existencia de dualidades al interior de la conciencia que determinan al Uno que se enfrenta al Otro. Dentro de la dualidad genérica sexual, los hombres detentan el lugar del Uno, universal y sujeto que define, mientras que las mujeres serían lo Otro, específico y objeto que es definido. Al interior de esta definición de roles, los hombres no tendrían necesidad de cuestionarse su posición, puesto que la sociedad es definida según su perspectiva; por otro lado, las mujeres, a través del feminismo y otras corrientes críticas, tienen el espacio para cuestionar desde su subjetividad su posición de Otro, especialmente si se considera que las explicaciones biologicistas no dan abasto para justificar las relaciones asimétricas entre los géneros. En este sentido, se han desarrollado una serie de teorías que permiten cuestionar las relaciones entre los géneros, especialmente desde una perspectiva del poder.

La concepción foucaultiana del poder permite aproximarse a la noción del género, ya que, además de otorgar a los individuos capacidades desiguales de afectar las conductas de un otro, éste también influye en los procesos de construcción de identidad de los individuos, en los cuales el género es un elemento esencial.

Para Foucault, es el poder el que produce sujetos y éstos no existen fuera de él $(2008 ; 2009)$. En el caso de la sexualidad, el autor indica que, desde el siglo XVIII, se ha establecido en torno a ella un discurso, basado en la racionalidad científica, que se comenzó a implementar desde ese momento (Foucault, 2008). Este discurso busca la producción y control de una sexualidad específica y útil al sistema capitalista burgués, asociada también a un sistema de construcción de saber y conocimiento en torno a la sexualidad. Cualquier aspecto que no obedezca a esta regulación se ve tildado de "perverso" y considerado como "patológico" en relación a la norma establecida. Un ejemplo de esto se puede observar en la patologización de la homosexualidad.

En esta misma línea de pensamiento se instala Butler (2002), quien plantea la existencia de la "matriz heterosexual". Ésta sería 
el conjunto de reglas simbólicas que se materializarían en la performance de los cuerpos en un ejercicio de poder, y cuya justificación estaría basada en las características biológicas de los sexos. Siendo la performance "una producción naturalizada y reiterada bajo presión mediante la fuerza de la prohibición y la amenaza del ostracismo" (Ibíd., p.145), la matriz generaría una concepción de la sexualidad binaria, produciendo cuerpos que performan lo masculino y femenino, los cuales se encuentran jerarquizados.

Por otro lado, también se establece una relación de poder entre los cuerpos que se ajustan en mayor manera a las exigencias de la matriz -es decir, los que se encuentran al interior de esta- $y$ aquellos cuerpos que se sitúan fuera de ella y que se establecen como objeto de temor y ejemplo de castigo para quienes no cumplen con la norma heterosexual.

La relación desigual de lo masculino por sobre lo femenino es un trabajo de construcción de lo simbólico que se imprime en los cuerpos por medio de la performance, pero también se extiende a las representaciones que los individuos hacen de esos cuerpos:

Los usos legítimos del cuerpo, sexuales sobre todo, que tiende a excluir del universo de lo sensible y de lo factible todo lo que marca la pertenencia al otro sexo, para producir ese artefacto social llamado hombre viril o mujer femenina. (Bourdieu, 2012, p.37)

Esta valorización de lo masculino por sobre lo femenino está presente también en los grupos LGBTI+, en las relaciones entre hombres homosexuales (Taywaditep, 2002) y en las relaciones entre hombres LGBTI+ y mujeres LGBTI+. En este último caso, las mujeres lesbianas estarían doblemente oprimidas, en su calidad de mujeres y en su calidad de lesbianas:

Las mujeres lesbianas sufren también de misoginia, y por lo tanto son doblemente afectadas, como mujeres y como lesbianas. Estos hechos están lejos de estar desvinculados: deben ser considerados como fuertemente conectados. Prueba de esto es que la comunidad de 
hombres gay no sufre tanto rechazo social y no es tan invisibilizado como la comunidad lésbica. Los hombres gay siguen siendo hombres, mientras que las mujeres lesbianas se enfrentan a un control y prejuicio doble. (Unanue, 2014, p.79)

Por otra parte, si bien tanto Foucault como Butler analizan el poder y las formas en que éste constriñe y crea sujetos, ambos conciben formas en que los sujetos pueden oponerse y resistirse al poder. Foucault indica que el poder solo se ejerce sobre sujetos libres, y "sólo en tanto ellos sean libres" (1988, p.16), es decir, sería el poder mismo el que genera instancias para su resistencia.

De manera similar, Butler, con su concepción de la matriz heterosexual y del sexo y género como construcciones sociales basadas en la norma heterosexual, indica que la matriz, al basarse en la repetición, no solo muestra su carácter arbitrario e impuesto, sino que también genera instancias en las que se puede romper con la performance exigida por la norma (Butler, 2002), puesto que la matriz, en su reiteración constante, puede fallar o se puede modificar de manera deliberada.

Si bien el género determina ciertos caminos a seguir en cuanto a performance y roles sociales que los sujetos han de actuar, también contiene en sí mismo instancias de subversión que dan espacio a la expresión de la subjetividad. En este sentido, el género sería un aspecto determinante de las experiencias de las mujeres lesbianas, que deben combinar las exigencias sociales con sus particularidades individuales.

\section{Género e identidades LGBTI+}

La división de género, como forma de organización y jerarquización social, se encuentra presente en una gran cantidad de sociedades, tanto premodernas como modernas (Bourdieu, 2012; Lamas, 1999). Ella ha puesto a la mujer en una situación marginal respecto del hombre en distintos contextos, ya sea la 
esfera pública, la privada, los espacios de trabajo, académicos, familiares, de ocio y tiempo libre, entre otros. Esta división establece ciertas reglas que configuran a la heterosexualidad como "lo normal", estigmatizando y penalizando todo aquello que se escape a ello (anormal). Esto se traduce en una doble discriminación contra las mujeres lesbianas en todas las esferas de la vida (Unanue, 2014; Villalba, 2008). Es por esto que el estudio del género, en su condición de dimensión que marca y forma las experiencias de los individuos en la sociedad, ha adquirido una importancia crucial.

En la década de los noventa, el estudio del género se complejizó con el desarrollo del concepto de "interseccionalidad", en el trabajo de la académica estadounidense Kimberlé Crenshaw (1989). Esta perspectiva considera la interacción entre distintas dimensiones de la vida de los individuos, como el género, la raza, clase o la sexualidad, y cómo estas interactúan entre sí, generando experiencias particulares en los individuos (Platero, 2013). Bajo esta mirada interseccional se han generado estudios sobre las intrarrelaciones de los grupos LGBTI+, que consideran las experiencias de distintos individuos miembros de estos grupos, dando cuenta de que distintas relaciones de género, raza, clase y sexualidad dan lugar a distintas experiencias de su sexualidad (Moore, 2012). Estas distintas experiencias implican no solo una diferencia, sino también una jerarquización de las mismas. De manera que las experiencias de los individuos LGBTI+ también se encuentran marcadas por las desigualdades que se observan a nivel general en la sociedad, generando una jerarquía de individuos dentro del mismo movimiento.

Se puede observar las relaciones que surgen entre raza, género y sexualidad de los individuos. Considerando el caso estadounidense, Moore indica que "la heterosexualidad negra se vuelve normativa mientras que la homosexualidad negra se convierte en desviada" (2012, pp.36-37). Esta distinción entre lo normativo y lo desviado genera una tensión en las identidades de las lesbianas negras, que deben balancear la idea de la respetabi- 
lidad negra (asociada a la clase media) con su autonomía de seres sexuados y, bajo el paradigma de la respetabilidad, desviados:

Las lesbianas negras movilizan identidades interseccionales y locaciones sociales en formas que les permiten mantener sus compromisos con su grupo racial, mientras que simultáneamente se presentan a sí mismas como seres sexualmente autónomos, aun cuando esa apertura de expresión de su sexualidad amenaza con perturbar supuestos de respetabilidad negra (de la clase media) particulares. (Moore, 2012, p.37)

Por otro lado, Todd (2004) analiza la jerarquía que se generaría al interior de grupos LGBT entre gays y lesbianas, por un lado, y bisexuales y personas trans por otro. Todd indica que la presencia de bisexuales y personas trans en el movimiento LGBTI+ es mal vista por gays y lesbianas, debido a que las vidas de personas bisexuales y trans generalmente no se ajustan al discurso del sujeto gay y lésbico "como tú", que busca los mismos ideales que otros individuos de la clase media estadounidense (a excepción de la pareja del mismo sexo). De forma similar Rosemblum (1994), desde la perspectiva del Derecho, hace un análisis de juicios en los que se han visto involucrados individuos LGB$\mathrm{TI}+$, dando cuenta de que el sistema legal estadounidense tiende a beneficiar a los individuos que el autor llama "excepto por", es decir, que se ajustan a las normas sociales o son ciudadanos modelo excepto por su sexualidad. En este sentido, se dejaría de lado la posibilidad de incorporación al sistema legal y de representación a sujetos LGBTI+ que no se ajusten del todo a las normas sociales hegemónicas, como lo son las personas de color o individuos pobres.

En América Latina, algunos estudios discuten con las nociones europeas y norteamericanas surgidas en torno a la sexualidad. Se cuestionan conceptos como "queer" y su aplicabilidad al contexto latinoamericano, criticando una aparente colonialidad conceptual (Foster, 2008). Además, se cuestiona la dicotomía 
gay/heterosexual: "los hombres que se comportan como 'verdaderos hombres' raramente sienten su sexualidad amenazada, y son relativamente libres de participar en inadvertidas prácticas homoeróticas privadas, y con frecuencia en cierta medida pública, con otros hombres" (Ibíd., p.934). Un último punto es la no consideración del género como categoría a incluir en las investigaciones respecto a los temas LGBTI+, estando el género "ausente como categoría a ser cuestionada, teorizada y deconstruidadesestructurada" (Ibíd., p.931), lo que deriva en la invisibilización de desigualdades o experiencias específicas estrechamente relacionadas con el género, así como también en una falta de cuestionamiento y crítica al concepto en sí mismo, lo que conlleva consecuencias que se traducen en la performance de los cuerpos, limitando y jerarquizando el actuar de los mismos.

\section{Feminismo, heterosexualidad y lesbianismo}

Si bien esta investigación aborda las experiencias y motivaciones de mujeres cis-género, hay que recordar que la identidad mujer o lesbiana no es una identidad monolítica, estática y definida, sino que es objeto de discusiones y múltiples concepciones. Parte de esta discusión se ha desarrollado al interior del feminismo en lo que se ha denominado "lesbofeminismo", donde se encuentran exponentes como Monique Wittig, Adrienne Rich o Judith Butler.

Una primera consideración es que "no es posible hablar de la mujer lesbiana como sujeto unitario y transcultural en todas las culturas, tiempos, sociedades y situaciones" (Kozak, 2011, p.159); en este sentido, como indica Kozak, no existe una identidad de mujer lesbiana predeterminada, sino que ésta está afectada por distintos factores, como la raza, la clase y los contextos sociales. Elaborando esta idea, y sobre la base de los planteamientos de Butler (2002), es posible indicar que la identidad de mujer lesbiana surge en torno a la matriz heterosexual que crea dos identidades jerarquizadas, masculinas y femeninas heterosexuales, pero 
que también genera identidades que no se adscriben a la matriz, como lo es la homosexualidad, y que quedarían aún más abajo en la jerarquía de identidades generada por esta matriz. De manera que la identidad de mujer lesbiana no es una característica ontológica de los sujetos, sino que es creada y adquiere relevancia en un sistema de poder específico, en este caso la matriz heterosexual. Este sistema, además, se relaciona con otros sistemas de poder y desigualdad, como son las clases sociales, la raza, el colonialismo, etc., que generan, en su interacción, distintas experiencias del ser mujer lesbiana.

Por otro lado, Adrienne Rich, en su texto La Heterosexualidad Obligatoria y la Existencia Lesbiana (1996), analiza la relación entre la sexualidad femenina y el poder masculino y cómo estos se configuran en la exigencia de una heterosexualidad obligatoria que invisibiliza las identidades que no se conforman a ella, como la identidad de la mujer lesbiana:

El supuesto de que "la mayoría de las mujeres son heterosexuales por naturaleza" es un muro teórico y político que bloquea al feminismo. (...) la heterosexualidad puede no ser en absoluto una "preferencia" sino algo que ha tenido que ser impuesto, gestionado, organizado, propagado y mantenido a la fuerza. (Rich, 1996, pp. 38-39)

Ahora bien, tanto la exigencia de la heterosexualidad femenina, que plantea Rich, como la construcción de una matriz heterosexual, que plantea Butler, se relacionan directamente con la jerarquización de los actos sexuales y, siguiendo a Rubin (como se cita en Moreno y Pichardo, 2006), con la "heteronormatividad", definida como aquellos "mecanismos sociales que canalizan o refuerzan la sexualidad de la mayoría de su población hacia la heterosexualidad" (p. 148). Esta "heteronorma" viene aparejada de una "homonormatividad", la cual produciría una jerarquización al interior de la comunidad LGBTI+ y una reproducción del sistema binario sexo/género, resultando en la invisibilización de ciertas identidades. Un ejemplo es la invisibilización de identi- 
dades como la bisexual o trans, siendo la primera considerada como una "identidad sexual que no acaba de encontrar su lugar" (Moreno et al., 2006, p. 149).

Así, la homosexualidad y otras identidades dicotómicas, "si bien ponen en cuestión la heteronormatividad del sistema sexo/ género, no ponen necesariamente en cuestión la construcción social del género, sino que en muchos casos incluso la reifican (...)" (Moreno et al., 2006).

Por último, Monique Wittig, quien plantea que las categorías de hombre y mujer, entre otras, solo existen al interior y debido al pensamiento heterosexual, siendo este un discurso con características universalizantes que oprime a lo que no se ajusta a él:

No puedo sino subrayar aquí el carácter opresivo que reviste al pensamiento heterosexual en su tendencia a universalizar inmediatamente su producción de conceptos, a formular leyes generales que valen para todas las sociedades, todas las épocas, todos los individuos (...) Así el lesbianismo, la homosexualidad, y la sociedad que podemos crear, no pueden ser pensadas o enunciadas, aunque siempre hayan existido. (Wittig, 2006, pp. 51-52)

El pensamiento heterosexual sería entonces un discurso de poder que influye y oprime las conductas de individuos, produciendo categorías y conceptos que le sean útiles, y rechazando las identidades que no se ajustan a él. Es en este contexto que Wittig afirma que "las lesbianas no son mujeres", en el sentido que la categoría mujer solo aparece y es funcional al interior del pensamiento heterosexual y, puesto que las lesbianas no participarían al interior de éste, no se puede pensar a la lesbiana como una mujer:

¿Qué es la mujer? Pánico, zafarrancho general de la defensa activa. Francamente es un problema que no tienen las lesbianas, por un cambio de Perspectiva, y sería impropio decir que las lesbianas viven, se asocian, hacen 
el amor con mujeres, porque "la mujer" no tiene sentido más que en los sistemas heterosexuales de pensamiento y en los sistemas económicos heterosexuales. Las lesbianas no son mujeres. (Wittig, 2006, p. 57)

Como se ve en las autoras anteriores, la heterosexualidad no sería una característica ontológica de los individuos, sino que se desarrolla como un ejercicio de poder que genera identidades y las jerarquiza. Por otro lado, habría ciertas diferencias en la concepción del ser lesbiana: entendiéndola como una especificidad del ser mujer, es decir, siendo posible hablar de la identidad de la mujer lesbiana, o rechazando una unidad entre ambos conceptos, como es el caso de Wittig, para quien las lesbianas no serían mujeres y no podría hablarse de una identidad de mujer lesbiana.

\section{Metodología}

La selección de este movimiento como caso de estudio radica en que existen orientaciones y objetivos generales compartidos por las distintas organizaciones, que hacen posible hablar de un solo movimiento. Temas como disminuir la desigualdad con base en la orientación sexual o identidad de género, y visibilizar las experiencias de personas LGBTI+ resultan claves. A pesar de esto, el cómo se busca cumplir los objetivos del movimiento puede variar entre organizaciones o entre individuos. Es por esto que se entrevistó a mujeres lesbianas participantes de cuatro organizaciones LGBTI+ distintas, que difieren entre sí en trayectoria, énfasis de objetivos, estructuras internas y perfiles de quienes las integran. Estas fueron: MOVILH, MUMS, Fundación Iguales y Rompiendo el Silencio. Específicamente, se realizaron entrevistas semiestructuradas -analizadas por medio de análisis de contenido temático (Bauer, 2000)- a diez mujeres pertenecientes a las organizaciones previamente mencionadas. 


\section{Resultados}

\section{Interseccionalidad e identidades en el movimiento LGBTI+}

En cuanto a la temática de interseccionalidad, abordada previamente en este artículo, destaca la relativa homogeneidad de las mujeres del movimiento. En este sentido, se observó que las mujeres entrevistadas, pertenecientes a organizaciones LGBTI+ de Santiago, no pertenecían a ninguna etnia indígena, todas tenían títulos universitarios o se encontraban en proceso de obtener uno, y se identificaban todas como lesbianas (no como bisexuales o alguna otra identidad de orientación sexual). Además, todas se identificaban a sí mismas como mujeres cis-género, es decir, que su género asignado al nacer coincide con el género con el que se identificaban tanto física como subjetivamente (Schilt \& Westbrook, 2009) al momento de realizadas las entrevistas.

Esta relativa similitud entre las entrevistadas no responde a una decisión metodológica previa al trabajo de campo, sino que es consecuencia de la conformación propia de las organizaciones a las que pertenecían, ya que ellas mismas comentaban que la participación de mujeres trans o bisexuales (por mencionar algunas) en sus organizaciones era baja. Sin embargo, la importancia de abordar las experiencias desde aquellas identidades es evidente y necesaria, por lo que investigaciones posteriores podrían ahondar en la temática de la caracterización de participantes del movimiento LGBTI+.

Estructura del movimiento: distintos medios, mismos objetivos

El movimiento LGBTI+ se constituye como tal al tener metas y objetivos en común, a pesar de las diferencias que puedan existir en los medios que las distintas organizaciones movilizan con el fin de alcanzarlos. Una de estas diferencias se observa en 
la división del trabajo al interior de la organización. En el caso particular de MOVILH y Rompiendo el Silencio, esta división es hecha a partir de la identidad de las personas que participan en ella. Así, en MOVILH existen distintos grupos, como "MOVILH Trans" y "MOVILH Mujeres", los cuales se encargan de levantar las actividades referidas a temas relacionados con esas identidades en particular. Mientras que en Rompiendo el Silencio esta distinción se refuerza mucho más, limitando la adhesión a la agrupación solo a quienes se identifican como mujeres:

Pero hombres, no. Ahí como que nosotras diferimos. Nosotras lo hemos hablado en el grupo y hay algunas que amenazan con irse. ¿Que nos brinden un servicio? Ni un problema, es decir, el que nos hace el diseño es hombre, el que nos ayudó con las redes sociales también, no es que estemos 'no, tiene que ser mujer', no. Pero para estar en el grupo, para estar en las reuniones y tener voz y voto, tiene que ser, obviamente, mujer. (Josefina ${ }^{5}$, Rompiendo el Silencio)

En el caso de las entrevistadas del MOVILH, existe una valoración de esta división a nivel de identidad:

Me parece que sí está bien dividir las tareas porque, por ejemplo, Álvaro no podría luchar por una causa de una mujer porque no es mujer, por lo tanto, puede ser empático, pero no puede estar un $100 \%$ comprometido o entender un $100 \%$ lo que yo quiero decir o yo quiero expresar. Ahora, que exista MOVILH-Mujer como... dentro de los grupos que podrían existir... o sea, MOVILH Trans y MOVILH... me parece bien porque hace que la mujer se salga un poco de la sombra del hombre. (Natalia, MOVILH)

\footnotetext{
5 Los nombres de las entrevistadas fueron reemplazados por pseudónimos para mantener su confidencialidad.
} 
Así, hay un esfuerzo en la estructura de la organización por visibilizar identidades que tienden a no ser consideradas en el imaginario de los movimientos LGBTI+, como son las mujeres lesbianas. Ahora bien, en lo que respecta a Fundación Iguales y MUMS, las entrevistadas identifican la ausencia del componente de género en la división logística de sus organizaciones, y que éste no es incorporado formalmente a la estructura de las mismas. Si bien existen esfuerzos por visibilizar ciertas identidades, estos se mantienen en el nivel de los/as participantes y no se constituyen como criterios para la existencia de grupos específicos de, por ejemplo, mujeres lesbianas o trans:

Sí estamos en la parada de visibilizar mucho más el trabajo que hagan las voluntarias que los voluntarios, sobre todo porque... no sé, nos ha pasado que los hombres... pareciera ser que tienen más redes de amigos, o más redes entre ellos (...). Entonces, tienen realidades mucho más conectadas que las mujeres. Eso hemos evidenciado. (Mariana, Fundación Iguales)

De esta manera, una diferencia importante entre las organizaciones del movimiento radicaría en cómo es incorporada la cuestión de género en sus estructuras formales y logísticas, existiendo algunas en las que se integra formalmente a la división organizacional realizada, como en el caso de MOVILH y Rompiendo el Silencio; mientras que en otras no se hace, como en Fundación Iguales y MUMS.

Esta diferencia de organización visibiliza el problema de la representatividad de identidades que no son la hegemónica (masculina homosexual cis-género) dentro del movimiento LGBTI+ en su conjunto. La existencia de instancias como Rompiendo el Silencio, "MOVILH Trans" o "MOVILH Mujeres", compuestas por sujetos cuyas identidades no coinciden con la hegemónica, permiten a estas identidades hablar desde un espacio formal, generando instancias para su representatividad en el movimiento y para levantar sus demandas particulares. Sin embargo, hay que 
recordar que la ausencia de la incorporación del género en las estructuras de las organizaciones, no implica que las mujeres lesbianas $-\mathfrak{u}$ otras identidades- al interior de ellas no tengan voz o capacidad de influencia, aunque esta se vea invisibilizada a nivel del imaginario que representa al movimiento.

\section{Motivación: desde la movilización de recursos hasta los sentimientos}

La motivación de las mujeres lesbianas para participar del movimiento LGBTI+ se puede dividir en dos momentos. El primero se relacionaría con aquello que incitó a las participantes a tomar la decisión de entrar a una organización perteneciente al movimiento LGBTI+, ya sea MUMS, Fundación Iguales, MOVILH o Rompiendo el Silencio. A su vez, esta motivación puede relacionarse con dos elementos importantes:

Mi polola me agarraba a mí, así como "cálmate" y yo así: “hueon, ¡me está agrediendo!". La tipa me quería pegar, (...) y la galla nos decía: "Mira, hay niños acá, ¿cómo es posible que ustedes hagan esto?...". Bueno, la cosa es que yo soy súper "parada" así que a mí me daba lo mismo ¿cachai? pero me quedé pensando que en verdad había sido un acto de discriminación. Y que no todo el mundo iba a reaccionar como yo (...) Entonces ahí yo dije: “Me voy a meter al MOVILH para ayudar a toda esta gente que no tiene como el carácter quizás como para salir adelante". (Isidora, MOVILH)

El primer elemento, como se observa en la cita anterior, tiene que ver con un suceso de discriminación particular (en este caso, contra la participante propiamente tal, pero también contra cercanos/as a ella) que lleva a las entrevistadas a tomar la decisión de trabajar por disminuir estos actos de discriminación, no solo en lo que refiere a su experiencia personal o la de su círculo cercano, sino que también para la comunidad LGBTI+. Estos epi- 
sodios se constituirán como aquellas "experiencias morales de menosprecio" planteadas por Honneth (1997) y que llevarían a las mujeres, al defraudar sus expectativas de reconocimiento, a querer hacer algo al respecto y participar del movimiento.

No obstante, la experiencia de discriminación en sí misma no se constituye como la única motivación de las mujeres por participar en el movimiento. Es aquí donde aparece un segundo elemento relacionado al primer momento de la motivación, que sería el sentimiento de responsabilidad moral para con la comunidad LGBTI+ en su conjunto, sentimiento que apuntaría a la realización de un trabajo en pos de mejorar sus condiciones de vida:

No queda otra cosa que dedicarse a eso, yo lo veo como una urgencia, no creo tener otra posibilidad, yo como mujer siento que no tengo otra posibilidad que dedicarme a esto. De verdad no puedo (...) No puedo. En inglés sería como compelled. Estoy como obligada a hacer esta cuestión, a nivel afectivo. Esto no es una cuestión que aparece ahora, el activismo en términos generales tiene que ver con una cuestión afectiva, con algo que te mueve a nivel tripa, a nivel corazón. Onda, tengo que hacerlo ¿cachai? (Bárbara, Fundación Iguales)

El inicio del trabajo en el movimiento LGBTI+ no responde a la mera persecución de metas y cumplimientos de ciertos objetivos -aunque ciertamente está contemplado-, sino que también se relaciona directamente con los sentimientos de las mujeres, con el deber que sienten, a nivel afectivo, de trabajar por la mejora de sus condiciones de vida. La responsabilidad moral referiría a una cuestión a "nivel corazón", a los sentimientos que tienen las mujeres, los que -como se vio en el marco teórico- pueden ir desde la ira hasta la solidaridad (Jasper, 1998).

Estos sentimientos, ya sean de solidaridad, indignación, deber moral o cualquier otro, no solo las movilizan a tomar la decisión de entrar al movimiento LGBTI+, sino que también las man- 
tiene dentro de él, en el trabajo constante. Esta permanencia se constituye como el segundo momento de la motivación:

Aparte porque son tus amigos, aparte en el MOVILH siempre tratamos de hacer un asado, y celebramos navidad, hacemos "amigo secreto", nos regalamos hueás, somos una familia. Ahora los chiquillos son mis amigos, o sea, me titulé, vamos a tomar $\mathrm{po}^{\prime}, \mathrm{y}$ vamos a tomar con ellos. (Isidora, MOVILH)

Los sentimientos de amistad, de cariño, de solidaridad con los compañeros y compañeras de la organización se constituyen como motivación para mantenerse dentro del movimiento. En este punto se evidencia la relación recíproca que tienen las motivaciones de las mujeres con su experiencia dentro del movimiento. Estos sentimientos refuerzan la mantención y la continuidad del trabajo, al mismo tiempo que están íntimamente relacionados con la experiencia que las mujeres tengan: el "hacer un asado" o cualquier otra actividad que genere lazos afectivos con los compañeros y compañeras generará aquellos sentimientos que llevan a las entrevistadas a quedarse.

La motivación de las mujeres lesbianas para mantenerse en el movimiento LGBTI+ también dependería de las ganas de generar un cambio, lo cual a su vez está relacionado con lo que se planteó acerca de los sentimientos de "deber moral" que movilizan a las entrevistadas:

Yo creo que hoy día es menos, y yo creo que la lucha que uno debe tener cuando ha hecho un camino que ya puede mirar hacia atrás, con lo que ya ha recorrido, es una obligación moral, ética, de compromiso para... que nadie pueda vivir lo que nosotros vivimos, los más viejos. Hasta tener que vivir una vida que no es lo tuyo, que jamás lo habrías hecho. (Francisca, Fundación Iguales)

Yo siento que tiene que haber un aporte real, en el sentido de que durante muchos años en este país se nos ha 
metido en la cabeza de que las cosas no se podían cambiar, que la gente se tenía que quedar callada, yo no creo en eso. No creo en los silencios. Tengo la convicción de que el mundo está para cambiarlo y es necesario generar esos cambios. (Valentina, MUMS)

Como se puede observar en ambas citas, la necesidad de cambios, de generar un antes y un después, es importante para las participantes, se constituye como un incentivo para el trabajo dentro de sus distintas organizaciones y, además, otorgaría un sentido más político a su participación en el movimiento. Además, este impulso por llevar adelante ciertos cambios generaría -una vez que esos cambios y objetivos son obtenidos- sentimientos de gratitud y satisfacción con el trabajo realizado, los que también se constituyen como motivantes para la continuación del trabajo que se realiza dentro del movimiento:

Yo tengo una amiga transexual en el MOVILH, sacó la bandera del MOVILH, y lo primero que les grita a los senadores es "ya no me van a pegar más, gracias" y todos los senadores así... y nosotros, llantos, todos... fue increíble, aplaudiendo, nos paramos como "ganamos" y no lo podías creer, o sea ganamos y la ley antidiscriminación es súper fuerte (...). Todo eso tú pensai que son pequeñas cosas, que fuiste dos o tres días, y te das cuenta que todas esas cosas que hiciste sirvió para que esta cuestión funcionara, entonces sentir eso de cuando se aprobó, fue emocionante, ahí llorando... todos llorando. (Isidora, MOVILH)

El caso particular de la intención de abandono de una entrevistada de su organización, si bien no se presenta en todas las narrativas, dadas las características de las entrevistadas (mujeres lesbianas pertenecientes a organizaciones del movimiento LGBTI+ al momento de la investigación), permite dar cuenta de que los sentimientos generados por la experiencia de las mujeres dentro del movimiento no solo son positivos y las mantienen al interior, sino que también pueden resultar ser negativos y tener como consecuencia el abandono. Sentimiento de injusticia, de 
que los medios que la organización moviliza no son los correctos, etc., adquieren importancia y se constituyen como razones para la desvinculación:

Desde la experiencia más personal, debido a los roces políticos, yo ahora me voy a retirar del MUMS. Básicamente porque no estoy de acuerdo con la política que se ha estado llevando a cabo, porque se dejó de hacer muchos estudios y MUMS se caracterizaba por levantar información, ¿cachai? (Valentina, MUMS)

De esta forma, aquellos sentimientos de descontento y disconformidad (Jasper, 1998), generados por las experiencias negativas que se vivieron dentro del movimiento, se constituyen como razones para distanciarse de éste.

Los sentimientos generados por las experiencias de las mujeres dentro de sus organizaciones, por sus expectativas de una sociedad mejor para la comunidad LGBTI+, inciden en lo que las mantiene en el trabajo. Como se examinó, la motivación y, por consiguiente, los medios que se movilizan en función de ésta, no puede remitirse netamente a la "acción racional", es decir, aquella que solo busca la conquista de ciertos objetivos. Por el contrario, éstos tienen un fuerte componente subjetivo y afectivo, en el que el cariño, la satisfacción, la felicidad, el descontento, e incluso el amor, se constituyen como aquello que mantiene a las participantes dentro del movimiento LGBTI+ y que, además, renueva el compromiso con el trabajo político.

\section{Experiencia: relación entre el sujeto y el sistema}

En el caso de la experiencia de las entrevistadas, la participación en una organización permite tener acceso a un espacio de desarrollo personal, en el cual, además, se generan lazos con otros participantes que se homologan a los de una familia. Se produce un intercambio por medio de la participación de las mujeres dentro del movimiento: entregan su esfuerzo y compromi- 
so, y reciben a cambio herramientas e instancias que les permiten afianzar la propia identidad como mujeres lesbianas, generando confianza en torno a ella:

A mí me ha servido más para afianzar mi condición, como a no tener miedo, antes uno igual tenía cierta reticencia a hablar del tema, uno así como... pasar bien piola, uno no hacía comentarios, nada, como que era. Y ahora como que ya digo perfectamente "mi polola", "mi novia", "soy lesbiana", como que no me interesa más que eso. Me ha ayudado un poco pertenecer a esta organización, como a afianzar esa identidad. (Natalia, MOVILH)

Cambia la forma de empoderarte, de ser mujer y de ser lesbiana, porque te da nuevas herramientas, te da una forma distinta de pararte frente al mundo y decir "sí, soy mujer, soy lesbiana". (Camila, Fundación Iguales)

Por otro lado, por medio de la participación en la organización, las entrevistadas desarrollan fuertes lazos con otros participantes. Sin embargo, estas relaciones, como indica Bertaux (2005), no son de carácter instrumental, sino que se configuran como relaciones afectivas y generadoras de sentido:

Los movimientos te van construyendo una otra familia que es con hermanos y hermanas de distintas edades y madres y padres que son igual que tú, eso es muy curioso, es como raro que te vean como la madre, la abuela, pero que sabes de lo que estás diciendo, no tienes que explicarle como yo se lo tuve que explicar a mi mamá. Es importante también. (Francisca, Fundación Iguales)

Estas relaciones y cambios personales de los que dan cuenta las entrevistadas y que se generan por medio de su experiencia en la organización, responden a la relación sujeto/sistema que plantea Dubet (2011) y a los significados que los sujetos le asignan a su participación en la organización (de Lauretis, 1989). En este caso, las entrevistadas informan de un cambio interno, en el 
sentido del empoderamiento y solidificación de su identidad de mujer lesbiana, y de la generación de lazos afectivos que ellas significan como una familia. Es decir, hay una relación entre la subjetividad de las entrevistadas y el espacio en el que se mueven, que se configura como lo externo a ellas pero que también las influencia. Además, los lazos afectivos generados y el crecimiento personal que experimentan las mujeres en su participación, producen -como se vio- sentimientos que las mantienen realizando un trabajo político, lo que da cuenta aún más de la relación recíproca entre sujeto y sistema.

\section{Experiencia y género: invisibilización de la identidad lésbica}

Considerando el género de las entrevistadas y cómo éste marca sus experiencias, se observan en sus narrativas temáticas como la invisibilidad de la mujer lesbiana y la universalidad de lo masculino, las distintas violencias que mujeres y hombres LGBTI+ sufren, y justificaciones de por qué se producen estos fenómenos.

En primer lugar, existe una tensión relacionada con la concepción que se tiene de los movimientos LGBTI+, mayormente masculinos, y las identidades de las entrevistadas, de mujeres lesbianas:

Yo estoy trabajando en "Iguales" hace muy poco. "Iguales" es una fundación conocida como la fundación de homosexuales con plata de clase alta, hombres. Entonces, ahí hay un desafío para mí directamente, porque yo... no, no soy hombre. Partiendo por ahí. Entonces, cómo me estai pidiendo que te hable desde ese lado, hay un desafío partiendo porque a mí sí me llegan... me llegan muchos comentarios sobre "a ver, ¿y dónde están las lesbianas?”. “¿Dónde están las chiquillas?”. “¿Dónde están las mujeres?". (Bárbara, Fundación Iguales)

Como se evidencia en la cita, las entrevistadas no solo deben lidiar con la discriminación presente en la sociedad en general, 
sino que también se enfrentan a una comunidad cuyo imaginario gira en torno a lo masculino, invisibilizando identidades distintas al hombre homosexual, lo que se constituye como la doble subordinación que experimentan las mujeres lesbianas. Frente a esta invisibilización, las mujeres generan discursos y justificaciones que tienden a asignarles la responsabilidad a las propias mujeres de su posición al interior del movimiento LGBTI+, sin problematizar su propia posición, tanto en la sociedad como al interior del movimiento:

Yo creo que aquí hay un fenómeno doble: no voy a decir un discurso victimizante de "nosotras pobres mujeres siempre estamos a la zaga, estamos a la retaguardia de los hombres que llevan la bandera". Aquí hay un problema fuerte de las lesbianas, que no les gusta visibilizarse ni empoderarse, (...) todas exigían, todas querían, todas necesitaban, pero cuando llegaba "el momento de los quiubos" necesitábamos gente para ir al Congreso, para ir al Parlamento, para ir a la marcha, nadie estaba dispuesta. (...) Con mujeres así de timoratas, temerosas, no podemos llevar el activismo lésbico a ninguna parte y eso se replica en toda clase social. (Teresa, Fundación Iguales)

Yo no sé por qué ninguna de nosotras es como la presidenta de "Iguales" o la directora de... MOVILH. Porque ahí está Ramón, el fundador es Rolando, pero las mujeres están en otra... a veces pienso que es por un tema de personalidad nuestra. De mujeres. (Francisca, Fundación Iguales)

Las justificaciones que las mujeres dan a su invisibilización le asignan a la mujer un actuar específico, el cual coincide con el rol de género socializado mediante la distinción binaria entre femenino/masculino. En este sentido, serían las características "más pasivas" de las mujeres las que dificultarían la consideración de las mismas en el imaginario y actuar del movimiento LGBTI+ 
chileno. Esto da cuenta, además, de que el desarrollo del movimiento LGBTI+ no puede separarse de la reproducción de los roles y relaciones de poder dictados por el imaginario heterosexual a través de la matriz.

Las experiencias de las mujeres lesbianas dan cuenta de una tensión entre sus identidades y las representaciones que existen al interior del movimiento, que valorizan lo masculino por sobre lo femenino. Sin embargo, las justificaciones que las entrevistadas otorgan a esta invisibilización no logran problematizar la reproducción de los roles del género al interior del movimiento, aunque sí identifican la existencia de una sociedad machista. Aquí se ejemplifica fuertemente la tensión entre lo subjetivo y lo externo de la cual habla Dubet (2011), y se observa cómo lo individual se encuentra fuertemente influenciado por las pautas sociales que dictan cómo deben actuar los sujetos, en este caso, estableciendo cómo deben performar los cuerpos generizados como "mujer".

\section{Experiencia y motivación: una relación recíproca}

Si bien analíticamente es posible separar motivación y experiencia, es importante recalcar que ambos conceptos se encuentran íntimamente relacionados en la realidad: la experiencia al interior del movimiento puede modificar las motivaciones para participar en él, ya sea reforzando el interés o disuadiendo a las entrevistadas de participar; al mismo tiempo que la motivación de las entrevistadas es un precedente para el inicio de su participación al interior de la organización y marca, además, las expectativas que ellas tengan frente a su experiencia al interior del movimiento.

En un caso de reforzamiento positivo se ve cómo las experiencias al interior del movimiento consolidan las motivaciones que puede tener una entrevistada:

Es que realmente siento que el MOVILH me apasiona caleta, porque tú te metes como voluntaria y en verdad tú estás haciendo nada, eres la encargada de cualquier 
cosa, de acarrear tonteras, y empezai a pensar cosas súper chicas, y con el tiempo te vas dando cuenta que en verdad las cosas no son tan así que en verdad que fueras a la fiesta, que fueras a ordenar cualquier cosa sirvió para que ahora la hueá fuera como... no sé po'. (...) te das cuenta que cuando se aprueba la ley, las personas empiezan a hablar, nos dimos cuenta que esto sirve y hay gente que viene y que nos apoya, y que hay gente que apoya, mucha gente que apoya, gente luchando por esto y de repente decís "pucha soy yo también la que está luchando por esto llevo años en esto" y que se aprobara esto es algo hermoso, es una hueá muy chica, pero ayudaste, y eso es... para mí es... Fue como el momento más emocionante en mi vida. (Isidora, MOVILH)

Por otro lado, las experiencias al interior de la organización pueden generar la desmotivación de la persona, lo que la lleva a querer terminar esa experiencia:

Lo que hace el Frente, por ejemplo, es hablar... "ya, esta marcha temática es de Ley de Identidad de Género". Eso es lo que se hace. Ahora, lamentablemente el MUMS, que sí estaba caracterizado por eso, ha decaído y ha caído en cuestiones muy performativas. $Y$ tiene que ver con que hubo un cruce generacional, de la antigua política, de quienes fundaron el movimiento con los nuevos que llegamos. Y quizás yo sentí que ya no hay cabida para levantar ese tipo de estudios. (Valentina, MUMS)

Así, tanto motivación como experiencia se encuentran relacionadas, puesto que hay una retroalimentación entre una y otra que se deriva de la relación entre lo subjetivo, los valores, la significación y las lógicas de acción que poseen los individuos, y el contexto en el que se desenvuelven, y si este contexto refuerza, modifica, o contradice las percepciones que tienen las entrevistadas de su participación en el movimiento LGBTI+ chileno. 


\section{Discusión}

La motivación de las mujeres para participar y mantenerse en el movimiento contempla los sentimientos generados por experiencias de discriminación sufridas tanto por las entrevistadas como por sus círculos cercanos, que pueden ir desde la ira, la impotencia, la compasión, entre otros. Aparejados a estos, habría también sentimientos de deber, de "obligación moral", que llevarían a las mujeres a desarrollar un trabajo político dentro de la organización para con la comunidad LGBTI+, teniendo como objetivo la mejora de sus condiciones de vida.

A su vez, para la mantención de las mujeres en este trabajo político, juegan un papel central sentimientos de cariño, afecto y alegría generados por la experiencia de las mujeres al interior de sus propias organizaciones y del movimiento en su conjunto. Aquí, la percepción de las compañeras y compañeros como "familia" es central, ya que se generan lazos de apoyo que refuerzan aún más estos sentimientos.

Por otro lado, a través de su experiencia en el movimiento LGBTI+, las mujeres adquieren nuevas significaciones acerca de su identidad y sobre los participantes del movimiento, en un proceso constante de significación. Cabe recordar que la relación entre la experiencia y las motivaciones de las mujeres es de constante reciprocidad, ya que las experiencias positivas generan sentimientos que se constituyen como motivaciones, impulsando el trabajo político de las entrevistadas.

En cuanto a la experiencia y su relación con el género de las entrevistadas, éstas se enfrentan a un escenario predominantemente masculino, tanto empírica como simbólicamente, y es sobre este escenario que las mujeres lesbianas deben negociar y buscar un espacio en el cual visibilizar su identidad. Sin embargo, las justificaciones que dan las mujeres a su posición desigual apuntan siempre a la responsabilidad que tienen ellas mismas de su propia invisibilización, sin problematizar la reproducción de roles que se da dentro del movimiento y la performance que 
les es asignada como mujeres al interior de la matriz heterosexual.

La necesidad de espacios dedicados exclusivamente a las mujeres es un ejemplo y expresión de la desigualdad de género al interior del movimiento LGBTI+ chileno, siguiendo la lógica de lo masculino/universal y femenino/específico. Aun cuando se crean espacios al interior de las organizaciones, no se generan políticas específicas para la inclusión de mujeres en los cargos directivos generales de la organización. De esta manera, aunque en ciertas organizaciones puede haber mujeres en estos cargos, no hay políticas internas que aseguren una representación femenina constante en los niveles más altos de la organización, lo que relega a las mujeres a participar solamente en los espacios dedicados a ellas (por ejemplo, "MOVILH Mujeres"). Esta subrrepresentación es también un reflejo de la reproducción de los roles de género asociados a la norma heterosexual y la distinción binaria femenino/masculino dentro del movimiento LGBTI+ y a nivel de sociedad en general, estando las mujeres lesbianas frente a una doble invisibilización de sus identidades al no performar acorde a la norma, lo que dificulta aún más su representatividad.

Por último, si bien estos hallazgos son importantes para la comprensión del movimiento LGBTI+ y también de la participación de los sujetos dentro de éste, se presentan nuevos desafíos que pueden ser abordados en futuras investigaciones. Aun cuando es clara la relación entre experiencias y la motivación por participar, podría surgir la pregunta acerca de cuál es el punto en que estas experiencias de menosprecio y discriminación se constituyen como motivación para el trabajo en un movimiento propiamente tal. En este sentido, ¿por qué personas LGBTI+ que también han sufrido estas experiencias se mantienen al margen del movimiento y sus organizaciones? ¿Qué es lo que hace la diferencia? Abordar estas preguntas en investigaciones futuras podría enriquecer aún más el conocimiento que se tiene sobre las motivaciones de los sujetos LGBTI+ para participar de un movimiento que busca reivindicar sus derechos. 


\section{Referencias}

Bauer, M. (2000). Classical Content Analysis: a Review, en M. W. Bauer \& G. Gaskell, (eds.), Qualitative Researching with text, image and sound: A Practical Handbook (pp.131-152). Londres: SAGE Publications.

Bertaux, D. (2005). Los Relatos de Vida. Perspectiva Etnosociológica. España: Bellaterra.

Bourdieu, P. (2012). La Dominación Masculina. España: Editorial Anagrama.

Butler, J. (2002). Cuerpos que Importan. Argentina: Editorial Paidós.

Cháneton, J. (2009). Género, Poder, y Discursos Sociales. Buenos Aires: Eudeba.

Crenshaw, K. (1989). Demarginalizing the intersection of race and sex: A black feminist critique of antidiscrimination doctrine, feminist theory and antiracist politics. U. of Chicago Legal Forum, 1, 139-167.

De Lauretis, T. (1989). Technologies of Gender Essays on Theory, Film and Fiction. Londres: MacMillan Press.

Dubet, F. (2011). La Experiencia Sociológica. España: Editorial Gedisa.

Foster, D. W. (2008). El estudio de los temas gay en América Latina desde 1980. Revista Iberoamericana, 74 (225), 923-941.

Foucault, M. (1988). El Sujeto y el Poder. Revista Mexicana de Sociología, $50(3), 3-21$.

Foucault, M. (2008). Historia de la Sexualidad Vol 1. (2 ed.). Argentina: Siglo Veintiuno Editores.

Foucault, M. (2009). Vigilar y Castigar. (2a ed.). España: Siglo XXI Editores.

Fundación Iguales. (s.f.). Nosotros/as. Fundación Iguales. Recuperado el 20 de noviembre de 2015, de http:/ / www.iguales.cl/nosotrxs/

Honneth, A. (1997). La Lucha por el Reconocimiento. Barcelona: Grijalbo.

Jasper, J. (1998). The Emotions of Protest: Affective and Reactive Emotions in and around Social Movements. Sociological Forum, 13 (3), 397-424.

Kirkwood, N. (2017). Feminarios. Buenos Aires: CLACSO. 
Kozak, G (2011). Estudio de las representaciones del sujeto mujer lesbiana. Mujeres Diversas. Miradas Feministas, 157.

Lamas, M. (1999) Usos, Dificultades y Posibilidades de la Categoría de Género. Papeles de Población, 5 (21), 147-178.

Latorre, M. (2005). Social Movements Beyond the Cultural Turn: Outline on the Rediscovery of Emotions. Politica y Sociedad, 42 (2), 37-48.

Melucci, A. (1999). Teoría de la acción colectiva. En A. Melucci, (ed.), Acción colectiva, vida cotidiana y democracia (pp. 25-54). México: El Colegio de México.

Moore, M. (2012). Intersectionality and the Study of Black, Sexual Minority Women. Gender E Society, 26 (1), 33-39.

Moreno, Á. \& Pichardo, J. I. (2006). Homonormatividad y existencia sexual. Amistades peligrosas entre género y sexualidad. AIBR. Revista de Antropología Iberoamericana, 1 (1).

Movimiento de Integración y Liberación Homosexual, MOVILH. (s.f.). Historia. MOVILH. Recuperado el 06 de abril de 2015 de http:/ /www.MOVILH.cl/quienes-somos/historia/

Movimiento por la Diversidad Sexual, MUMS. (s.f.) Historia. MUMS. Recuperado el 23 de noviembre de 2015, de http:/ /www. mums.cl/quienes-somos/historia/

Platero, R. L. (2013). Marañas con distintos acentos: género y sexualidad en la perspectiva interseccional. Encrucijadas-Revista Crítica de Ciencias Sociales, 5, 44-52.

Rich, A. (1996). Heterosexualidad obligatoria y existencia lesbiana. DUODA: Estudis de la diferència sexual, (10), 15-48.

Robles, V. H. (2008). Bandera Hueca: Historia del Movimiento Homosexual de Chile. Santiago: Editorial Cuarto Propio.

Rosenblum, D. (1994). Queer Intersectionality and the Failure of Recent Lesbian and Gay "Victories". Law E Sexuality, 84, 25-55.

Schilt, K. \& Westbrook, L. (2009). Doing Gender, Doing Heteronormativity: "Gender Normals," Transgender People, and the Social Maintenance of Heterosexuality. Gender \& Society, 23 (4), 440-464.

Taywaditep, J. (2002). Marginalization Among the Marginalized. Journal of Homosexuality, 42 (1), 1-28.

Todd, J. (2004). GL vs. BT: The Archaeology of Biphobia and Transpho- 
bia Within the U.S. Gay and Lesbian Community. Journal of Bisexuality, 3, 25-55.

Unanue Cuesta, M. C. (2014). Social Exclusion and the Stigmatization of Lesbians. Procedia - Social and Behavioral Sciences, 161, 77-81.

Villalba, P. (2008). ¿Techo de cristal o armario de doble fondo? Análisis de discurso sobre el lesbianismo y la homosexualidad desde una perspectiva de género, en R. Platero (ed.), Lesbianas Discursos y Representaciones (pp. 139-172). España: Editorial Melusina.

Wittig, M. (2006). El pensamiento heterosexual, en M. Wittig, El pensamiento heterosexual y otros ensayos. Barcelona: Egalés. 